\title{
Long-term clinical outcome of acute myocardial infarction according to the early revascularization method: a comparison of primary percutaneous coronary interventions and fibrinolysis followed by routine invasive treatment
}

\author{
Hyang Ki Min, Ji Young Park, Jae Woong Choi, Sung Kee Ryu, Seunghwan Kim, \\ Chang Sup Song, Dong Shin Kim, Chi Woo Song, Se Jong Kim, Young Bin Kim \\ Division of Cardiology, Department of Internal Medicine, Eulji General Hospital, Seoul, Korea
}

\begin{abstract}
Background: This study was conducted to provide a comparison between the clinical outcomes of primary percutaneous coronary intervention $(\mathrm{PCl})$ and that of fibrinolysis followed by routine invasive treatment in ST elevation myocardial infarction (STEMI).

Methods: A total of 184 consecutive STEMI patients who underwent primary $\mathrm{PCl}$ or fibrinolysis followed by a routine invasive therapy were enrolled from 2004 to 2011, and their major adverse cardiovascular events (MACEs) were compared.

Results: Among the 184 patients, 146 patients received primary PCl and 38 patients received fibrinolysis. The baseline clinical characteristics were similar between both groups, except for triglyceride level $(68.1 \pm 66.62$ vs. $141.6 \pm 154.3 \mathrm{mg} / \mathrm{dL}, p=0.007)$ and high density lipoprotein level $(44.6 \pm 10.3 \mathrm{vs} .39 .5 \pm 8.1 \mathrm{mg} / \mathrm{dL}, p=0.005)$. The initial creatine kinase-MB level was higher in the primary PCl group $(71.5 \pm 114.2$ vs. $35.9 \pm 59.9 \mathrm{ng} / \mathrm{mL}$, $p=0.010)$. The proportion of pre-thrombolysis in Ml 0 to 2 flow lesions $(92.9 \%$ vs. $73.0 \%, p<0.001)$ was higher and glycoprotein $\mathrm{Ilb} / \mathrm{llla}$ inhibitors were administered more frequently in the primary $\mathrm{PCl}$ group. There was no difference in the 12-month clinical outcomes, including all-cause mortality $(9.9 \%$ vs. $8.8 \%, p=0.896)$, cardiac death $(7.8 \%$ vs. $5.9 \%, p=0.845)$, non-fatal MI $(1.4 \%$ vs. $2.9 \%, p=0.539)$, target lesion revascularization $(5.7 \%$ vs. $2.9 \%, p=0.517$ ), and stroke ( $0 \%$ vs. $0 \%$ ). The MACEs free survival rate was similar for both groups (odds ratio, $0.792 ; 95 \%$ confidence interval, $0.317-1.980 ; p=0.618$ ). The clinical outcome of thrombolysis was not inferior, even when compared with primary $\mathrm{PCl}$ performed within 90 minutes.

Conclusion: Early fibrinolysis with optimal antiplatelet and antithrombotic therapy followed by appropriate invasive procedure would be a comparable alternative to treatment of $\mathrm{Ml}$, especially in cases of shortersymptom-to-door time.
\end{abstract}

Keywords: Fibrinolysis; Percutaneous coronary intervention; Myocardial infarction

Received: August 19, 2016, Revised: August 9, 2017

Accepted: August 10, 2017

Corresponding Author: Sung Kee Ryu, Division of Cardiology, Department of Internal Medicine, Eulji General Hospital, Eulji University, 14 Hangeolbiseok-Gil, Nowon-gu, Seoul 01830, Korea

Tel: +82-2-970-8671, Fax: +82-2-970-8887

E-mail: ysk1140@eulji.ac.kr

*Hyang Ki Min and Ji Young Park equally contributed to this work. First author: Hyang Ki Min and Ji Young Park

\section{INTRODUCTION}

Early reperfusion is a key paradigm in the current treatment method for acute phase ST-segment-elevation myocardial infarction (STEMI). Current guidelines recommend primary percutaneous coronary intervention (PCI) as the preferred reperfusion strategy over fibrinolytic therapy in STEMI [1-3]. However, timely primary $\mathrm{PCI}-$ as recommended by

Copyright (C) 2017 Yeungnam University College of Medicine

This is an Open Access article distributed under the terms of the Creative Commons Attribution Non-Commercial License (http://creativecommons.org/licenses/by-nc/4.0/) which permits unrestricted non-commercial use, distribution, and reproduction in any medium, provided the original work is properly cited. 
the guidelines (door-to-balloon or door-to-instrument time $\leq 90$ minutes) - may not always be available, especially at night. Facilitated PCI is defined as a planned PCI after the administration of medical therapy, such as fibrinolytic therapy or glycoprotein IIb/IIIa (GpIIb/IIIa) inhibitors, and it is supposed to be a good alternative to primary PCI, improving the early coronary artery patency with a reduction of thrombotic burden and myocardial salvage [4]. However, according to the Assessment of the Safety and Efficacy of a New Treatment Strategy with Percutaneous Coronary Intervention (ASSENT$4 \mathrm{PCI}$ ) trial and meta-analysis, this method was associated with worse clinical outcome; therefore, planned facilitated PCI after fibrinolysis is not recommended [3,5,6]. Sub-analysis from the ASSENT-4 PCI trial showed that facilitated PCI had better a pre-procedural thrombolysis in myocardial infarction (TIMI) when compared with the primary PCI group, but stent thrombosis increased in the facilitated PCI group due to prothrombotic condition after thrombolytic therapy [7]. However, implementation of dual antiplatelet therapy, subcutaneous enoxaparin injection, routine thrombus aspiration, more fibrin specific fibrinolysis agent, PCI with new generation drug eluting stent, and closing device are expected to change the results. In the real world, routine invasive methods such as coronary angiography and PCI are commonly performed on the same day after thrombolysis, even in patients whose symptoms have subsided. Therefore, the present study was conducted to evaluate whether the clinical outcome or lesion-related complications, such as stent thrombosis or restenosis, would be significantly higher in thrombolysis followed by a routine invasive strategy. Moreover, we analyzed particular clinical circumstances that induce thrombolytic therapies that are as effective as primary PCI.

\section{MATERIALS AND METHODS}

A total of 184 consecutive acute STEMI patients underwent primary PCI or fibrinolytic therapy with tenecteplase (Metalyze) followed by elective PCI on the same day, between January 2004 and December 2011 at Eulji general hospital. Of the 184 patients, 146 patients received primary PCI and 38 patients received fibrinolysis. In the primary PCI group, patients who underwent cardiopulmonary resuscitation during PCI or on admission were excluded.

Patients were eligible for enrollment if they presented with- in 3 hours of the onset of symptoms, had no evidence of acute STEMI on their qualifying electrocardiogram (at least $2 \mathrm{~mm}$ in two contiguous peripheral or precordial leads), and could not undergo primary PCI within 1 hour of the first medical contact. Patients who received tenecteplase along with antiplatelet and anticoagulant therapy underwent coronary angiography within 6 to 24 hours of fibrinolysis. Rescue coronary intervention was performed there was less than 50\% ST-segment resolution in the single lead with maximum elevation or clinical evidence of failed reperfusion within 90 minutes of fibrinolysis. All patients provided written informed consent.

All patients received a $300 \mathrm{mg}$ aspirin loading dose and a $300 \mathrm{mg}$ to $600 \mathrm{mg}$ clopidogrel loading dose before PCI, followed by daily administration of $75 \mathrm{mg}$ clopidogrel, which was recommended to be continued for at least 1 year. PCI was performed via the femoral approach, and hemostasis of the puncture site was conducted using a closing device. As an antithrombotic therapy for PCI, all patients received 60 mg enoxaparin (Clexane) $60 \mathrm{mg}$ before and after PCI during the hospital stay (within 7 days), and unfractionated heparin $50 \mathrm{U} / \mathrm{kg}$ prior to PCI for the first hour. Tenecteplase was administered in a weight-based dose $(30 \mathrm{mg}$ if the weight was 55 to $<60 \mathrm{~kg}, 35 \mathrm{mg}$ if the weight was 60 to $<70 \mathrm{~kg}, 40 \mathrm{mg}$ if the weight was 70 to $<80 \mathrm{~kg}, 45 \mathrm{mg}$ if the weight was 80 to $<90 \mathrm{~kg}$, and $50 \mathrm{mg}$ if the weight was $\geq 90 \mathrm{~kg}$ ) and was combined with low-molecular-weight enoxaparin $(30 \mathrm{mg}$ intravenous bolus followed by subcutaneous injection of $1 \mathrm{mg}$ per kilogram of body weight $[0.75 \mathrm{mg}$ per kilogram for patients $\geq 75$ years of age] every 12 hours) except for patients 75 years of age or older, in whom the intravenous bolus was omitted. In cases with no-reflow or visible thrombi, adjunctive GPIs were administered at the discretion of the primary physician.

Diagnostic angiography and PCI were performed through either the femoral or radial artery after the administration of unfractionated heparin $(70 \mathrm{U} / \mathrm{kg}$ to $100 \mathrm{U} / \mathrm{kg})$. Patients received unfractionated heparin to maintain the activated clotting time of $>250 \mathrm{~s}$ during the procedure. Stents were deployed after a prior balloon angioplasty, and the use of cilostazol or glycoprotein inhibitors was left to the discretion of the individual operator.

Rather than utilizing the artheroablative devices, a simple pre-dilation was performed to obtain an adequate luminal 
diameter, which was necessary to accommodate the unexpanded drug eluting stents and their delivery system. Thrombus aspiration was performed in patients who had visible thrombi or slow distal flow (TIMI 0-2) after ballooning or stenting.

Successful PCI was defined as the achievement of an angiographic residual stenosis $<30 \%$ in the presence of TIMI blood flow grade 3. During the in-hospital period, patients received medical treatment, including beta-blockers, angiotensin conver ting enzyme inhibitors or angiotensin II receptor blockers, calcium channel blockers, and statins. After discharge, patients were encouraged to continue the same medication they were receiving in the hospital, except for intravenous or temporary medications.

We compared the major adverse cardiovascular events (MACEs) during a 12-month period between the two groups, which included all-cause mortality, non-fatal reinfarction, target lesion revascularization, and any stroke. We also evaluated their long-term clinical outcomes and survival rates.

All statistical analyses were performed using SPSS 18.0 (SPSS Inc., Chicago, IL, USA) Continuous variables were expressed as the mean \pm standard deviation and compared using a Student's t-test. Cox proportion hazard regression analysis was used to evaluate the survival rate.

Categorical data were expressed as percentages and compared using chi-squared statistics or Fisher's exact test. A $p$-value of $<0.05$ was considered statistically significant.

To adjust for potential confounders, propensity score matching analysis was conducted using the logistic regression model, which tested the propensity to have facilitated PCI after fibrinolysis treatment in STEMI patients rather than primary PCI. We tested all available variables that could be of potential relevance; namely, age, sex, symptom to door time, peak troponin $\mathrm{T}$, low density lipoprotein, diabetes mellitus, hypertension, smoking, and left ventricular ejection fraction. The logistic model by which the propensity score was estimated showed the predictive value well $(\mathrm{C}$-static $=0.648)$.

\section{RESULTS}

The baseline clinical characteristics showed that age was similar in both groups $(60.5 \pm 11.7$ vs. $57.0 \pm 12.2$ years, $p=$ $0.111)$, the initial levels of creatine kinase-MB (CK-MB) were higher in the primary PCI group $(71.5 \pm 114.2$ vs. $35.9 \pm 59.9$ $\mathrm{ng} / \mathrm{mL}, p=0.010$ ), and triglyceride levels were higher in the fibrinolysis group (68.1 \pm 66.6 vs. $141.6 \pm 154.3 \mathrm{mg} / \mathrm{dL}, p=$ 0.007). Previous medications, including aspirin, clopidogrel, beta blocker, and lipid lowering agents, were similar between groups (Table 1). Angiographic characteristics showed that the pre-TIMI 0 to 2 flow lesion $(92.9 \%$ vs. $73.0 \%, p<0.001)$ and the use of GpIIb/IIIa inhibitors (35.6\% vs. $15.8 \%, p=$ $0.019)$ were higher in the primary PCI group. The proportion of post-TIMI flow grade 3 was similar between groups (85.3 vs. $94.1 \%, p=0.17$ ) (Table 2 ). There was no difference in the 12-month clinical outcome, including all-cause mortality ( $9.9 \%$ vs. $8.8 \%, p=0.896)$, cardiac death $(7.8 \%$ vs. $5.9 \%, p=$ $0.845)$, non-fatal MI (1.4\% vs. $2.9 \%, p=0.539)$, target lesion revascularization $(5.7 \%$ vs. $2.9 \% p=0.517)$, and stroke $(0 \%$ vs. 0\%) (Table 3). Age (odds ratio [OR], 1.054; 95\% confidence interval [CI], 1.003-1.106) and current smoking (OR, 3.954; 95\% CI, 1.085-14.409) were significant independent risk factors for 1-year MACE, but thrombolysis (OR, 0.696; 95\% CI, 0.189-2.566) and primary PCI were not, which was in accordance with the multiple regression analysis after an adjustment of various clinical factors (Table 4). The median follow-up period was 1,650 days (1,653 vs. $1,562.5$ days), and there was no significant difference in the long-term clinical outcome between groups (Table 5). Cox proportional multiple regression analysis for MACE free survival, adjusting for other clinical, anatomical, and procedural parameters, also showed no significant clinical difference between the two groups (Fig. 1, 2).

We also analyzed the differences in clinical outcomes between primary $\mathrm{PCI}$ with a door to balloon time $\leq 90$ minutes and fibrinolysis followed by coronary angiography. The difference in clinical features was similar to the whole cohort. There was no significant difference in the clinical features, except for the initial CK-MB level. In the fibrinolysis group, symptom-to-door time was shorter without statistical significance. Multivariable Cox regression analysis showed that the facilitated PCI after fibrinolysis had similar incidences of total death, cardiac death, recurrent non-fatal MI, TLR, stent thrombosis, and MACEs than primary PCI. The adjusted clinical outcomes at 1 year also showed that the facilitated PCI after fibrinolysis had similar incidences of total death, cardiac death, recurrent non-fatal MI, TLR, stent thrombosis, and MACEs (Table 6). There was no significant difference in the 1-year clinical outcome (Table 7) or long-term MACE free survival between groups. The curve comparing the MACE 
Hyang Ki Min et al.

Table 1. Baseline clinical characteristics

\begin{tabular}{|c|c|c|c|}
\hline & Primary PCI $(n=146)$ & Fibrinolysis $(\mathrm{n}=38)$ & $p$-value \\
\hline Male & $116(79.5)$ & $29(76.3)$ & 0.673 \\
\hline Age (years) & $60.5 \pm 11.7$ & $57.0 \pm 12.2$ & 0.111 \\
\hline Diabetes mellitus & $35(24.0)$ & $9(23.7)$ & 0.970 \\
\hline Hypertension & $62(42.5)$ & $14(36.8)$ & 0.531 \\
\hline Current smoking & $63(43.2)$ & $18(47.4)$ & 0.641 \\
\hline Cerebrovascular disease & $3(2.1)$ & $0(0)$ & 0.373 \\
\hline Total cholesterol & $186.9 \pm 35.9$ & $194.2 \pm 36.3$ & 0.265 \\
\hline Triglyceride & $68.1 \pm 66.62$ & $141.6 \pm 154.3$ & 0.007 \\
\hline $\mathrm{HDL}(\mathrm{mg} / \mathrm{dL})$ & $44.6 \pm 10.3$ & $39.5 \pm 8.1$ & 0.005 \\
\hline $\mathrm{LDL}(\mathrm{mg} / \mathrm{dL})$ & $125.8 \pm 31.5$ & $130.2 \pm 33.7$ & 0.454 \\
\hline Initial CK-MB (ng/mL) & $71.5 \pm 114.2$ & $35.9 \pm 59.9$ & 0.010 \\
\hline Initial Troponin $(\mathrm{ng} / \mathrm{mL})$ & $1.7 \pm 3.6$ & $0.9 \pm 2.0$ & 0.073 \\
\hline Peak CK-MB (ng/mL) & $235.6 \pm 60.6$ & $204.1 \pm 160.0$ & 0.283 \\
\hline Peak Troponin (ng/mL) & $7.5 \pm 6.6$ & $8.5 \pm 7.7$ & 0.432 \\
\hline Creatinine $(\mathrm{mg} / \mathrm{dL})$ & $1.1 \pm 0.4$ & $1.1 \pm 0.4$ & 0.408 \\
\hline LV ejection fraction (\%) & $50.6 \pm 9.3$ & $51.3 \pm 9.1$ & 0.700 \\
\hline Previous use of aspirin & $12(8.2)$ & $3(7.9)$ & 0.948 \\
\hline Previous use of clopidogrel & $9(6.2)$ & $2(5.3)$ & 0.835 \\
\hline Previous use of beta blocker & $2(1.4)$ & $1(2.6)$ & 0.584 \\
\hline Previous use of lipid lowering agent & $4(2.7)$ & $2(5.3)$ & 0.435 \\
\hline Symptom to door time (min) & $245.4 \pm 286.1$ & $173.5 \pm 241.0$ & 0.157 \\
\hline Symptom to door time (min, median) & 131 & 87.5 & \\
\hline Door to balloon time (min, median) & 95 & & \\
\hline Door to needle time (min, median) & & 46.5 & \\
\hline Needle to device time ${ }^{\mathrm{a})}$ (hours, median) & & 8.7 & \\
\hline
\end{tabular}

Values are presented as mean \pm standard deviation and number (\%).

a) Time from thromboysis to admission to cardiac catheterization room. PCI, percutaneous coronary intervention; HDL, high-density lipoprotein; LDL, low-density lipoprotein; CK-MB, creatine kinase-MB; LV, left ventricular.

free survival between groups is shown in Fig. 1. The hazard ratio for fibrinolysis followed by PCI was 0.792 (95\% CI, $0.317-1.980 ; p=0.618)$ after adjusting for age, sex, symptomto-door time, peak troponin T, triglyceride, diabetes, hypertension, and smoking (Fig. 1). Comparison of MACE free survival between PCI (DBT $\leq 90$ minutes) and fibrinolysis followed by CAG was shown in Fig. 2. The hazard ratio for fibrinolysis followed by PCI was 0.729 (95\% CI, 0.256-2.075; $p=0.554)$ after adjusting for age, sex, symptom-to-door time, peak troponin $\mathrm{T}$, triglycerides, diabetes, hypertension, and smoking (Fig. 2).

\section{DISCUSSION}

Based on our analysis, early fibrinolysis followed by routine invasive therapy was not inferior to primary PCI. Most of our recruited patients were collected prior to 2010, and the regulation for door-to-balloon time within 90 minutes was not strongly adapted. Therefore, this cohort includes many cases in which the door-to-balloon time was greater than 90 minutes. However, when compared with patients who received primary PCI within 90 minutes, fibrinolysis followed by routine invasive treatment was not inferior. The fibrinolysis group had a higher proportion of multi-vessel disease and unfavorable lipid profiles. Nevertheless, the initial CK-MB was significantly lower and symptom onset to the first medical contact time was shorter without statistical significance.

Our analysis showed that the primary PCI group had more frequently used GpIIb/IIIa inhibitors when compared with the fibrinolysis group. However, the proportion of post TIMI 


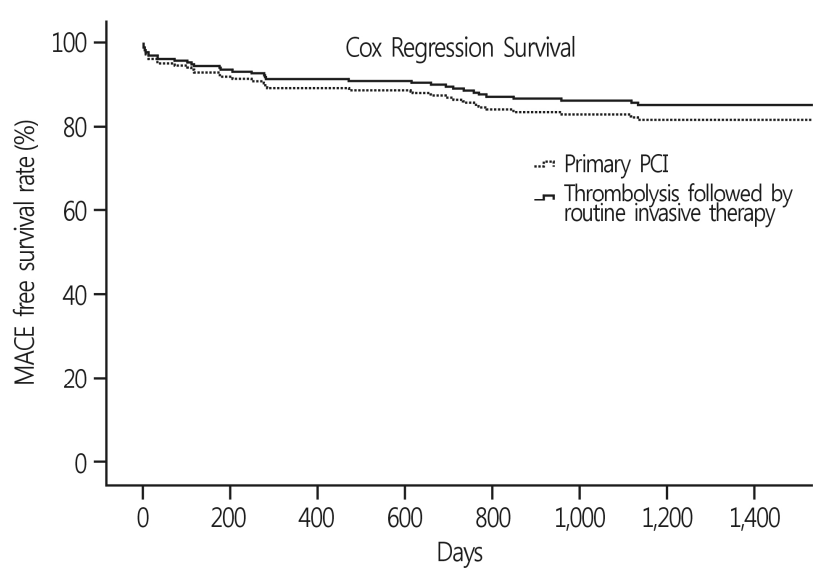

Fig. 1. Comparison of MACE free survival curve between the two groups. The hazard ratio for fibrinolysis followed by PCI was 0.792 (95\% CI, 0.317-1.980; $p=0.618$ ) after adjusting for age, sex, symptom-to-door time, peak troponin $\mathrm{T}$, triglyceride, diabetes, hypertension, and smoking. MACE, major adverse cardiovascular events; HR, hazard ratio; PCI, percutaneous coronary intervention; $\mathrm{CI}$ : confidence interval.

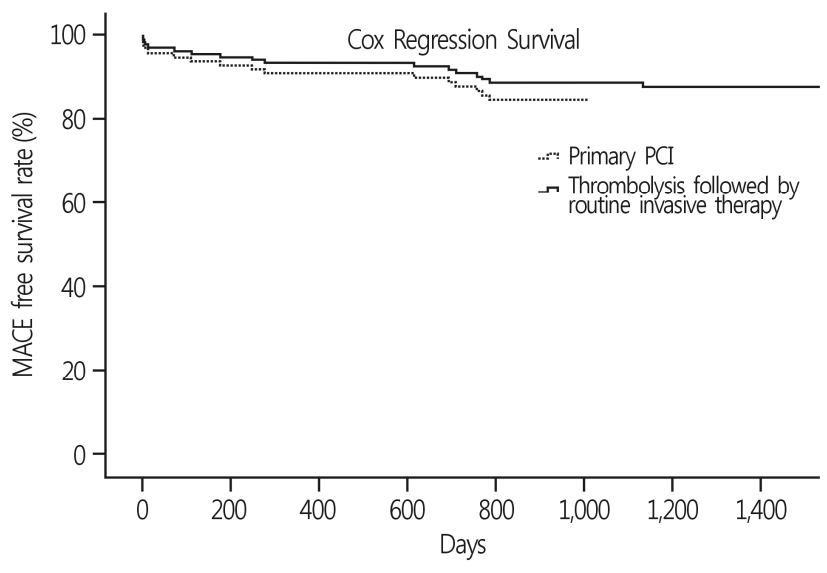

Fig. 2. Comparison of MACE free survival curve between PCI (DBT $\leq 90$ minutes) and fibrinolysis followed by CAG. The hazard ratio for fibrinolysis followed by PCI was 0.729 (95\% CI, 0.256$2.075 ; p=0.554)$ after adjusting for age, sex, symptom-to-door time, peak troponin $\mathrm{T}$, triglyceride, diabetes, hypertension, and smoking. MACE, major adverse cardiovascular events; HR, hazard ratio; PCI, percutaneous coronary intervention; CI, confidence interval; DBT, door to balloon time; CAG, coronary angiography.

Table 2. Baseline angiographic and procedural characteristics

\begin{tabular}{|c|c|c|c|}
\hline Variable & Primary PCI $(n=146)$ & Fibrinolysis $(\mathrm{n}=38)$ & $p$-value \\
\hline \multicolumn{4}{|l|}{ Infarct-related artery } \\
\hline Left Main PCI & $3(2.1)$ & $0(0.0)$ & 0.373 \\
\hline LAD PCI & $54(37.0)$ & $20(52.6)$ & 0.080 \\
\hline LCX PCI & $13(8.9)$ & $2(5.3)$ & 0.465 \\
\hline RCA PCI & $48(32.9)$ & $12(31.6)$ & 0.879 \\
\hline Calcified lesions & $26(18.6)$ & $6(15.8)$ & 0.692 \\
\hline Stent diameter $(\mathrm{mm})$ & $3.4 \pm 0.5$ & $3.6 \pm 7.0$ & 0.094 \\
\hline Stent length $(\mathrm{mm})$ & $19.9 \pm 5.6$ & $19.5 \pm 5.0$ & 0.720 \\
\hline \multicolumn{4}{|c|}{ Severity of coronary artery disease } \\
\hline $1 \mathrm{VD}$ & $80(54.8)$ & $11(28.9)$ & \\
\hline $2 \mathrm{VD}$ & $47(32.2)$ & $18(47.4)$ & \\
\hline $3 \mathrm{VD}$ & $19(13.0)$ & $9(23.7)$ & 0.016 \\
\hline Left main & $5(3.4)$ & $1(2.6)$ & 0.806 \\
\hline \multicolumn{4}{|c|}{ Pre-PCI TIMI flow grade (review was available in 177 patients) } \\
\hline $0-2$ & $130(92.9)$ & $27(73.0)$ & \\
\hline 3 & $10(7.1)$ & $10(27.0)$ & 0.001 \\
\hline \multicolumn{4}{|c|}{ Post-PCI TIMI flow grade } \\
\hline $0-2$ & $20(14.7)$ & $2(5.9)$ & \\
\hline 3 & $116(85.3)$ & $32(94.1)$ & 0.170 \\
\hline \multicolumn{4}{|c|}{ GpIIb/IIIa inhibitor and thrombectomy } \\
\hline Aspiration & $57(39.0)$ & $11(28.9)$ & 0.251 \\
\hline GpIIb/IIIa inhibitor & $52(35.6)$ & $6(15.8)$ & 0.019 \\
\hline
\end{tabular}

Values are presented as mean \pm standard deviation and number (\%).

PCI, percutaneous coronary intervention; LAD, left anterior descending branch; LCX, left circumflex artery; RCA, right circumflex artery; VD, vessel disease; TIMI, thrombolysis in myocardial infarction; GpIIb/IIIa, glycoprotein IIb/IIIa. 
3 flow and cumulative incidence of MACE for up to 12 months were similar between the two groups.

In 2007, the American College of Cardiology and the American Heart Association guidelines commented that "facilitated

Table 3. Clinical outcomes up to 1 year

\begin{tabular}{lccc}
\hline \multicolumn{1}{c}{ Variable } & $\begin{array}{c}\text { Primary PCI } \\
(\mathrm{n}=136)\end{array}$ & $\begin{array}{c}\text { Fibrinolysis } \\
(\mathrm{n}=34)\end{array}$ & $p$-value \\
\hline Total death & $14(9.9 \%)$ & $3(8.8 \%)$ & 0.896 \\
Cardiac death & $11(7.8)$ & $2(5.9)$ & 0.845 \\
Recurrent non-fatal MI & $2(1.4)$ & $1(2.9)$ & 0.539 \\
TLR & $8(5.7)$ & $1(2.9)$ & 0.517 \\
Stroke & $0(0.0)$ & $0(0.0)$ & NS \\
MACE & $19(13.5)$ & $4(11.8)$ & 0.791 \\
\hline
\end{tabular}

Values are presented as mean \pm standard deviation and number (\%). Follow-up was lost in nine patients (primary PCI, five; fibrinolysis, four) in 1 year.

PCI, percutaneous coronary intervention; MI, myocardial infarction; TLR, target lesion revascularization; MACE, major adverse cardiovascular events.
PCI using regimens other than full-dose fibrinolytic therapy might be considered as a reperfusion strategy when all of the following are present: (a) patients are at high risk, (b) PCI is not available within 90 minutes, and (c) bleeding risk is low; younger age, absence of poorly controlled hypertension, nor-

Table 4. Predictors for 1-year major adverse cardiovascular events in multiple binary logistic regression analysis

\begin{tabular}{lclc}
\hline Variable & OR & \multicolumn{1}{c}{$95 \%$ CI } & $p$-value \\
\hline Age & 1.054 & $1.003-1.106$ & 0.036 \\
Male sex & 3.537 & $0.921-13.579$ & 0.066 \\
Symptom to door time & 1.001 & $0.999-1.002$ & 0.309 \\
Peak troponin T & 1.058 & $0.987-1.134$ & 0.113 \\
Diabetes & 2.275 & $0.810-6.387$ & 0.119 \\
Hypertension & 0.935 & $0.337-2.590$ & 0.897 \\
Current smoking & 3.954 & $1.085-14.409$ & 0.037 \\
Facilitated PCI after fibrinolysis & 0.696 & $0.189-2.566$ & 0.586 \\
\hline
\end{tabular}

Values are presented as number (\%).

$\mathrm{OR}$, odds ratio; CI, confidence interval; PCI, percutaneous coronary intervention.

Table 5. Overall long-term clinical outcome

\begin{tabular}{lccc}
\hline Variable & Primary PCI $(\mathrm{n}=146)$ & Fibrinolysis $(\mathrm{n}=38)$ & $p$-value \\
\hline FU duration (median, days) & 1,653 & 1,562 & $1,442 \pm 874$ \\
FU duration (mean, days) & $1,714 \pm 1,139$ & $3(7.9)$ & 0.173 \\
All-cause death & $24(16.6)$ & $3(7.9)$ & 0.180 \\
Cardiac death & $11(7.8)$ & $3(7.9)$ & 0.949 \\
Recurrent non-fatal MI & $9(6.2)$ & $2(5.3)$ & 0.700 \\
TLR & $12(8.2)$ & $0(0.0)$ & 0.517 \\
Stent thrombosis & $5(3.4)$ & $0(0.0)$ & 0.247 \\
Stroke & $5(3.4)$ & $6(15.8)$ & 0.247 \\
MACE & $39(26.7)$ & 0.163 \\
\hline
\end{tabular}

Values are presented as mean \pm standard deviation and number (\%).

PCI, percutaneous coronary intervention; FU, follow up; TLR, target lesion revascularization; MI, myocardial infarction; MACE, major adverse cardiovascular events.

Table 6. Adjusted cumulative clinical outcomes at the 1-year mark of facilitated percutaneous coronary intervention after fibrinolysis compared with primary percutaneous coronary intervention (Cox Regression Analysis Using Propensity Score)

\begin{tabular}{lcccc}
\hline Variable & Unadjusted OR (95\% CI) & $p$-value & Adjusted OR (95\% CI) & $p$-value \\
\hline All-cause death & $0.43(0.12-1.52)$ & 0.191 & $0.59(0.16-2.19)$ & 0.435 \\
Cardiac death & $1.04(0.27-3.94)$ & 0.949 & $1.33(0.34-5.26)$ & 0.678 \\
Recurrent non-fatal MI & $1.05(0.27-3.97)$ & 0.940 & $1.04(0.26-4.04)$ & 0.951 \\
TLR & $0.62(0.13-2.89)$ & 0.544 & $0.62(0.13-2.99)$ & 0.559 \\
Stent thrombosis & $0.95(0.10-8.84)$ & 0.971 & $0.88(0.09-8.51)$ & 0.917 \\
MACE & $0.51(0.20-1.32)$ & 0.168 & $0.69(0.18-2.56)$ & 0.586 \\
\hline
\end{tabular}

Values are presented as number (\%).

OR, odds ratio; CI, confidence interval; TLR, target lesion revascularization; MI, myocardial infarction; MACE, major adverse cardiovascular events. 
mal body weight (Level of Evidence: C)". Moreover, the guidelines warned that planned reperfusion strategies employing full-dose fibrinolytic therapy followed immediately by PCI could have adverse effects [3]. The ASSENT-4 PCI trial is the key study supporting the guidelines to not recommend planned facilitated PCI. However, this trial has some limitations. Specifically the authors commented that the optimum antithrombotic co-therapy could not be conducted in the facilitated PCI arm. Because of concern regarding bleeding complications, which might attenuate the benefits of early reperfusion with tenecteplase, infusion of heparin after bolus and up-front loading of clopidogrel with GPI was prohibited, except in bail-out case [6. They also pointed out that the symptom-to-fibrinolysis time might be too long (median 2.6 hours) to obtain the maximum effects of tenecteplase. Another randomized trial compared primary PCI with facilitated PCI with half dose fibrinolysis and facilitated PCI with only the GpIIb/ IIIa inhibitors. In that study, antiplatelet and anticoagulation therapies were optimally given to all groups, and there was no significant difference in the 90-day mortality [8].

A recently published randomized trial comparing primary PCI and fibrinolysis followed by routine invasive treatment also showed a different result from the ASSENT-4 PCI trial. A total of 1,892 STEMI patients, who were admitted within three hours of the symptom onset and could not receive primary PCI in the first hour because of admittance were randomly assigned to either primary PCI within one hour or fibrinolysis with tenecteplase, clopidogrel, and enoxaparin before being transported to PCI-capable hospitals. Coronary angiography was conducted within 6-24 hours of fibrinolysis and PCI was conducted in cases of incomplete revascularization.

Table 7. Comparison of baseline characteristics and 1-year clinical outcome between percutaneous coronary intervention with door to balloon time $\leq 90$ minutes and fibrinolysis followed by routine invasive therapy

\begin{tabular}{|c|c|c|c|}
\hline Variable & Primary PCI $(n=68)$ & Fibrinolysis $(n=38)$ & $p$-value \\
\hline Male & $55(80.9)$ & $29(76.3)$ & 0.578 \\
\hline Age (years) & $59.6 \pm 11.7$ & $57.0 \pm 12.2$ & 0.111 \\
\hline Diabetes mellitus & $15(22.1)$ & $9(23.7)$ & 0.848 \\
\hline Hypertension & $24(35.3)$ & $14(36.8)$ & 0.873 \\
\hline Current smoking & $29(42.6)$ & $18(47.4)$ & 0.639 \\
\hline Total cholesterol & $186.8 \pm 39.0$ & $194.2 \pm 36.3$ & 0.337 \\
\hline Triglyceride & $68.8 \pm 79.8$ & $141.6 \pm 154.3$ & 0.009 \\
\hline $\mathrm{HDL}(\mathrm{mg} / \mathrm{dL})$ & $43.8 \pm 10.4$ & $39.5 \pm 8.1$ & 0.030 \\
\hline $\mathrm{LDL}(\mathrm{mg} / \mathrm{dL})$ & $125.6 \pm 33.5$ & $130.2 \pm 33.7$ & 0.496 \\
\hline Initial CK-MB (ng/mL) & $72.9 \pm 120.6$ & $35.9 \pm 59.9$ & 0.038 \\
\hline Initial Troponin $(\mathrm{ng} / \mathrm{mL})$ & $1.55 \pm 2.9$ & $0.9 \pm 0.0$ & 0.159 \\
\hline Peak CK-MB (ng/mL) & $228.2 \pm 164.4$ & $204.1 \pm 160.0$ & 0.467 \\
\hline Peak Troponin (ng/mL) & $7.2 \pm 6.6$ & $8.5 \pm 0.7$ & 0.464 \\
\hline Creatinine (mg/dL) & $1.1 \pm 0.4$ & $1.1 \pm 0.4$ & 0.300 \\
\hline Severity of CAD & & & 0.008 \\
\hline $1 \mathrm{VD}$ & $40(58.8)$ & $11(28.9)$ & \\
\hline $2 \mathrm{VD}$ & $22(32.4)$ & $18(47.4)$ & \\
\hline $3 \mathrm{VD}$ & $6(8.8)$ & $9(23.7)$ & \\
\hline Stent diameter & $3.42 \pm 0.52$ & $3.63 \pm 0.67$ & 0.118 \\
\hline Symptom to door time (min) & $233.2 \pm 291.7$ & $173.5 \pm 241.0$ & 0.286 \\
\hline 1 year MACE & $8(12.1)$ & $4(11.8)$ & 0.959 \\
\hline 1 year non fatal $\mathrm{MI}$ & $2(3.0)$ & $1(2.9)$ & 0.980 \\
\hline 1 year all-cause mortality & $7(10.6)$ & $3(8.8)$ & 0.778 \\
\hline 1 year TLR & $3(4.5)$ & $1(2.9)$ & 0.698 \\
\hline
\end{tabular}

Values are presented as mean \pm standard deviation and number (\%).

PCI, percutaneous coronary intervention; DBT, door to balloon time; HDL, high-density lipoprotein; LDL, low-density lipoprotein; CK-MB, creatine kinase-MB; CAD, coronary artery disease; VD, vessel disease; MACE, major adverse cardiovascular events. 
In the STREAM trial, they reported that composite of death, congestive heart failure, shock, and re-infarction were similar. The rates of stroke were low in the two study groups, but both intracranial hemorrhagic and primary ischemic strokes were more frequent in the fibrinolysis group than the primary PCI group. Following reduction of the dose of tenecteplase in patients 75 years of age or older, there were no cases of intracranial hemorrhage ( 0 of 97 patients), while there this occurred in 3 of 37 patients (8.1\%) in this age group before the amendment. The rate of major non-intracranial bleeding was $6.5 \%$ in the fibrinolysis group, while it was $4.8 \%$ in the primary PCI group, a difference that was not significant ( $p=$ 0.11). The rates of blood transfusions were also similar in the two study groups (2.9\% and 2.3\%, respectively; $p=0.47)$.

The STREAM trial suggests that if immediate primary PCI is difficult, fibrinolysis with full antiplatelet and anticoagulation therapy followed by a routine invasive strategy within one day might be a good alternative for primary PCI.

Our study population is more similar to that of the STREAM trial than that of ASSENT-4 PCI. Moreover, PCI was not performed immediately as was done in ASSENT-4 PCI (median of 104 minutes), but was instead conducted within 6-24 hours and followed by optimal antiplatelet and antithrombotic therapy. PCI immediately after fibrinolysis is more likely to be exposed to a more prothrombotic environment, which might lead to worse clinical outcomes [7,9].

Although fibrinolysis is currently considered a second line therapy for early revascularization in STEMI, many clinical studies have confirmed that it still has an important role in some instances. For example, if prompt primary $\mathrm{PCI}$ is not available, fibrinolysis is recommended, especially, if the primary PCI-related time delay is over 120 minutes. Pre-hospital fibrinolysis to reduce ischemic time was also attempted and its effectiveness on in-hospital fibrinolysis was reported by meta-analysis [10]. Gershlick et al. commented that if primary PCI was compared with prehospital fibrinolysis, the result might be different [11]. Second, an open coronary artery does not always mean recovery of the coronary artery because primary PCI is not a simple opening procedure and requires complex drug therapy and other mechanical procedures with high skill. Thus, the results of primary PCI cannot always be the same or predicted.

We summarized the reasons for a comparable clinical result in fibrinolysis as follows. After fibrinolysis with proper in- vasive treatment, the residual significant vascular lesion was treated properly. A previous study reported a thrombotic problem in PCI following fibrinolysis; however, in our analysis, thrombotic complications were not significant. This may be because of recent advancements in the antiplatelet and antithrombotic methods, i.e., use of the 2 nd generation drug eluting stents. Moreover, invasive treatment was also conducted within one day of fibrinolysis, but it was not conducted promptly as in the ASSENT-4 PCI trial to avoid paradoxical thrombotic crisis after fibrinolysis. The stent size was also slightly larger in the fibrinolysis group. Because of the thrombotic burden and vascular spastic reaction in an acute stage of STEMI, proper sizing of the stent or balloon is difficult, even with an intravascular ultrasound. Proper stent sizing might improve the clinical outcomes in the fibrinolysis group. An earlier start of treatment might be an important factor for this result. Finally, in our analysis all fibrinolysis patients received tenecteplase (Metalyze) as the thrombolytic agent, which is known to be the most fibrin-specific agent, and to cause less major bleeding complications, such as hemorrhagic stroke [12].

It should be noted that this study had several limitations. First, although there was no significant difference in major risk factors, it was not a randomized study. However, the proportions of dyslipidemia and multiple diseases were higher in fibrinolysis; thus, a comparable result in the fibrinolysis group cannot be due to the favorable baseline clinical characteristics. Furthermore, primary PCI after cardiopulmonary resuscitation or patients who showed cardiac arrest or severe hemodynamic derangement during primary PCI were excluded from our analysis. Second, in this study, patients were enrolled over 7 years, but the number of patients in each arm was significantly less than the number for the duration. The reason that the number of patients in each group is small considering the period as the limitation of study conducted in single center. Finally, the most important limitation of this study was that there were no data describing bleeding events and hemorrhagic stroke, which are major side effects of thrombolytic therapy.

To the best of our knowledge, this study is the first to report a comparable prognosis between fibrinolysis followed by a routine invasive therapy and primary PCI in a Korean population. We did not expect a comparable result between fibrinolysis and primary PCI, or a result that suggested the 
effectiveness of routine facilitated PCI. However, in the event that a timely primary PCI is not available, early fibrinolysis with optimal antiplatelet and antithrombotic therapy followed by an appropriate invasive procedure may be a good alternative strategy, especially in cases in which there is a shorter symptom-to-door time.

\section{CONFLICT OF INTEREST}

No potential conflict of interest relevant to this article was reported.

\section{ORCID}

Hyang Ki Min, https://orcid.org/0000-0002-5883-234X

Ji Young Park, https://orcid.org/0000-0002-6097-059X

Sung Kee Ryu, https://orcid.org/0000-0003-1673-0166

\section{REFERENCES}

1. Carville S, Harker M, Henderson R, Gray H; Guideline Development Group. Acute management of myocardial infarction with ST-segment elevation: summary of NICE guidance. BMJ 2013;347:f4006.

2. Task Force on the management of ST-segment elevation acute myocardial infarction of the European Society of Cardiology (ESC); Steg PG, James SK, Atar D, Badano LP, BlömstromLundqvist C, et al. ESC Guidelines for the management of acute myocardial infarction in patients presenting with STsegment elevation. Eur Heart J 2012;33:2569-619.

3. Antman EM, Hand M, Armstrong PW, Bates ER, Green LA, Halasyamani LK, et al. 2007 focused update of the ACC/AHA 2004 guidelines for the management of patients with ST-elevation myocardial infarction: a report of the American College of Cardiology/American Heart Association Task Force on Practice Guidelines: developed in collaboration with the Cana- dian Cardiovascular Society endorsed by the American Academy of Family Physicians: 2007 writing group to review new evidence and update the ACC/AHA 2004 guidelines for the management of patients with ST-elevation myocardial infarction, writing on behalf of the 2004 Writing Committee. Circulation 2008;117:296-329.

4. Capodanno D, Dangas G. Facilitated/pharmaco-invasive approaches in STEMI. Curr Cardiol Rev 2012;8:177-80.

5. Keeley EC, Boura JA, Grines CL. Comparison of primary and facilitated percutaneous coronary interventions for ST-elevation myocardial infarction: quantitative review of randomised trials. Lancet 2006;367:579-88.

6. Assessment of the Safety and Efficacy of a New Treatment Strategy with Percutaneous Coronary Intervention (ASSENT4 PCI) investigators. Primary versus tenecteplase-facilitated percutaneous coronary intervention in patients with ST-segment elevation acute myocardial infarction (ASSENT-4 PCI): randomized trial. Lancet 2006;367:569-78.

7. Zalewski J, Bogaerts K, Desmet W, Sinnaeve P, Berger P, Grines C, et al. Intraluminal thrombus in facilitated versus primary percutaneous coronary intervention: an angiographic substudy of the ASSENT-4 PCI (Assessment of the Safety and Efficacy of a New Treatment Strategy with Percutaneous Coronary Intervention) trial. J Am Coll Cardiol 2011;57:1867-73.

8. Ellis SG, Tendera M, de Belder MA, van Boven AJ, Widimsky $\mathrm{P}$, Janssens L, et al. Facilitated PCI in patients with ST-elevation myocardial infarction. N Engl J Med 2008;358:2205-17.

9. Collet JP, Montalescot G, Le May M, Borentain M, Gershlick A. Percutaneous coronary intervention after fibrinolysis: a multiple meta-analyses approach according to the type of strategy. J Am Coll Cardiol 2006;48:1326-35.

10. Morrison LJ, Verbeek PR, McDonald AC, Sawadsky BV, Cook DJ. Mortality and prehospital thrombolysis for acute myocardial infarction: a meta-analysis. JAMA 2000;283: 2686-92.

11. Gershlick AH, Banning AP, Myat A, Verheugt FW, Gersh BJ. Reperfusion therapy for STEMI: is there still a role for thrombolysis in the era of primary percutaneous coronary intervention? Lancet 2013;382:624-32.

12. Melandri G, Vagnarelli F, Calabrese D, Semprini F, Nanni S, Branzi A. Review of tenecteplase (TNKase) in the treatment of acute myocardial infarction. Vasc Health Risk Manag 2009; 5:249-56. 